\title{
Comparison of Open-Source CFD Software for Aerodynamic Analysis of Mini-UAV
}

Tomas Bata University in Zlin

Faculty of Applied Informatics

\section{Tomáš Vogeltanz}

Department of Informatics and Artificial Intelligence

Tomas Bata University in Zlín

Czech Republic 


\section{Content

- Free Software

- Mini-UAVs

- Aerodynamic Analysis 
- The aerodynamic analysis often plays a major role in the early stage of a design phase.

- Major changes during conceptual phase

- Minor changes after conceptual phase

- Incorrect analysis => loss of money

- Suitable aerodynamic behavior is necessary.

- Two options:

- Wind tunnel test

- CFD analysis 


\section{Free Software}

\section{Design}

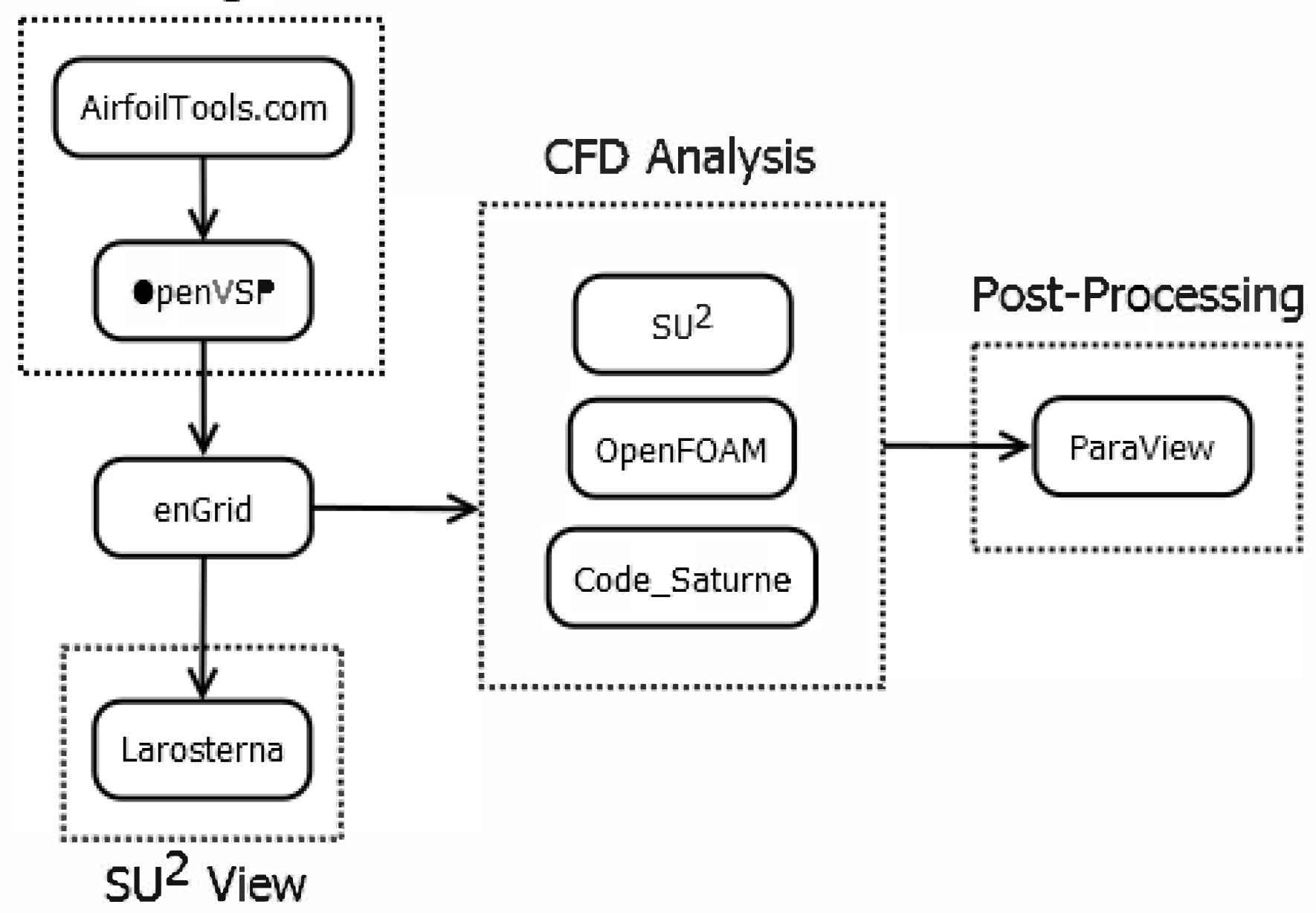

Figure 1. Free Software Connections 
Design

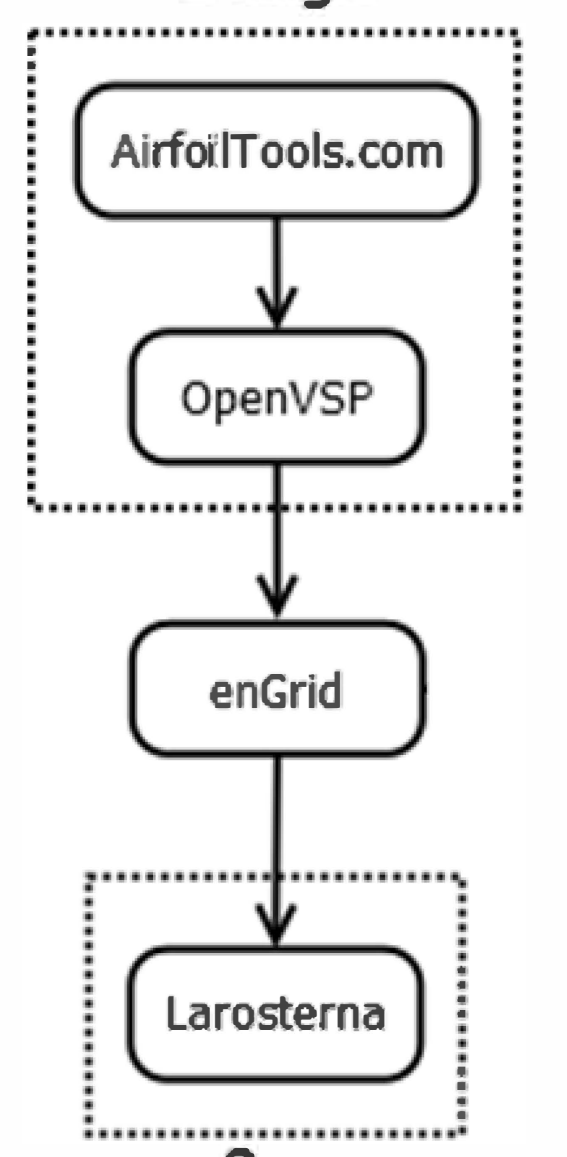

$\mathrm{SU}^{2}$ View
- OpenVSP is a parametric and easyto-use aircraft geometry application.

- AirfoilTools generates the Selig and Lednicer airfoil DAT files.

- enGrid is an open-source mesh generation application.

- Larosterna is a design tool with an option to import a SU2 mesh file.

Figure 2. Design \& Mesh 
CFD Analysis

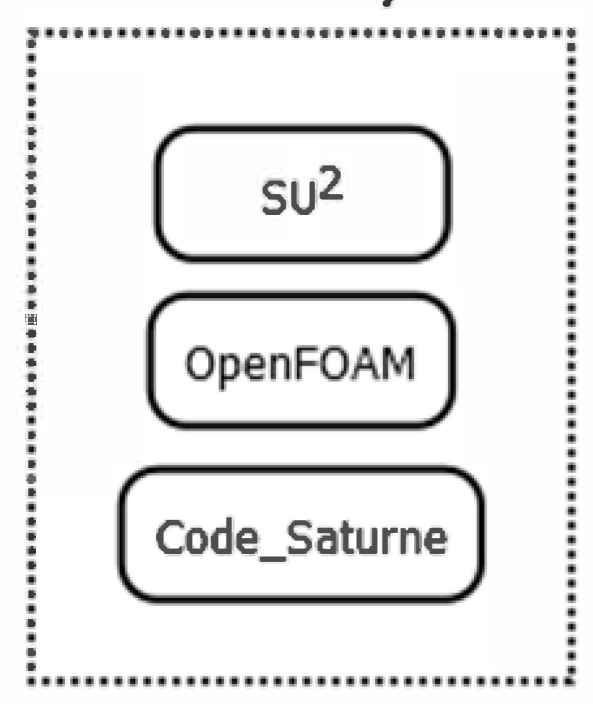

Figure 3. CFD Software
- The Stanford University Unstructured $\left(\mathbf{S U}^{2}\right)$ suite solves complex, multiphysics analysis and optimization tasks using arbitrary unstructured meshes. 
CFD Analysis

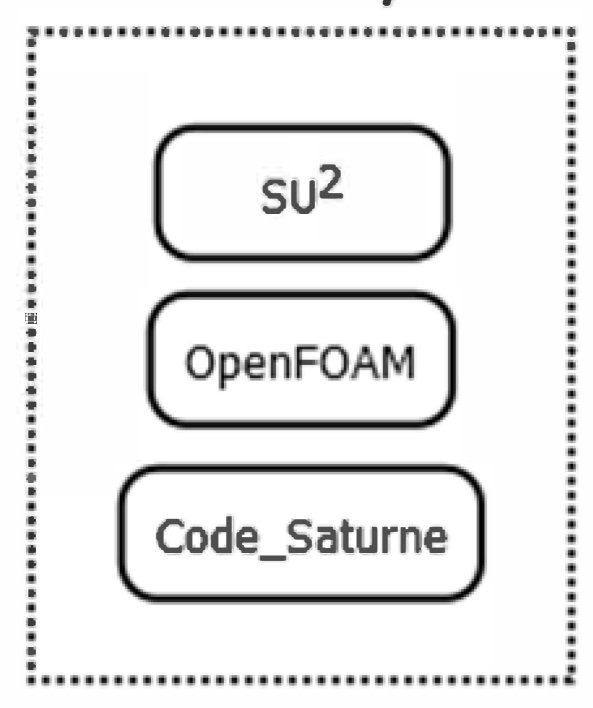

Figure 3. CFD Software
- The Open Field Operation and Manipulation (OpenFOAM) CFD Toolbox includes:

- over 80 solver applications

- over 170 utility applications for pre- and post-processing tasks 


\section{CFD Analysis}

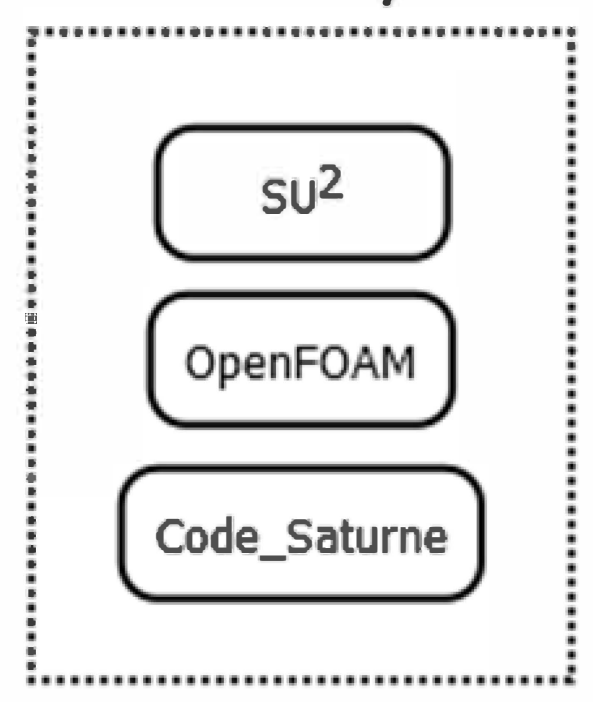

Figure 3. CFD Software
- Code_Saturne is based on a Finite Volume Method which accepts 3D meshes built with

- any type of cell (tetrahedral, hexahedral, prismatic, pyramidal, and polyhedral)

- any type of grid structure (unstructured, block structured, hybrid) 
- Common parameters of mini-UAVs:

- Wingspan < $6 \mathrm{~m}$

- Weight $<25 \mathrm{~kg}$

- Low speeds (between 20 and $120 \mathrm{~km} / \mathrm{h}$ )

- Low altitudes (from 3 to $1000 \mathrm{~m}$ )

- Primary Requirements:

- Long flight duration

- All-weather capabilities

- Success in missions 


\section{SAGITTA}

Tomas Bata University in Zlin

Faculty of Applied Informatics

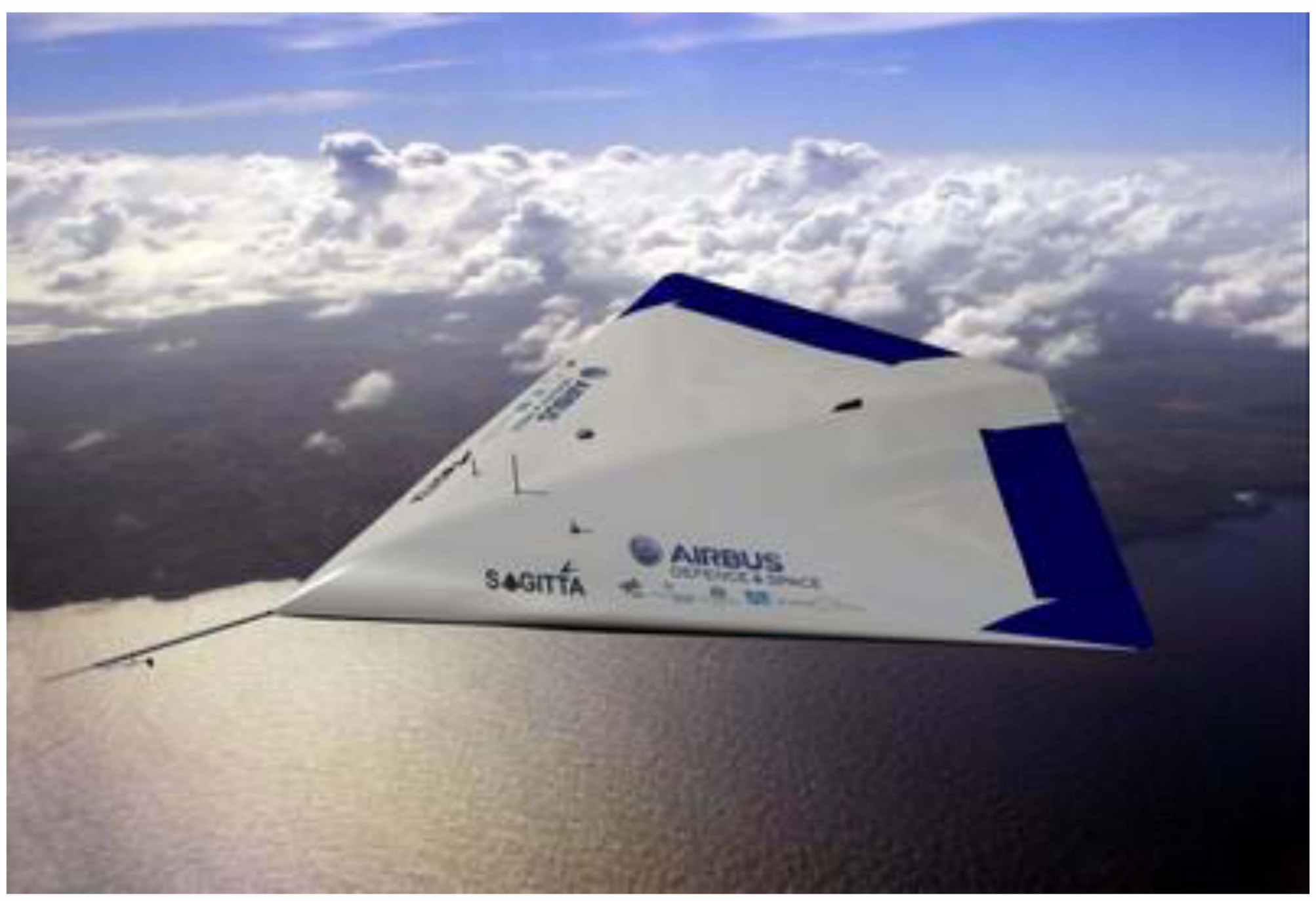

Figure 4. SAGITTA UCAV Concept [1] 


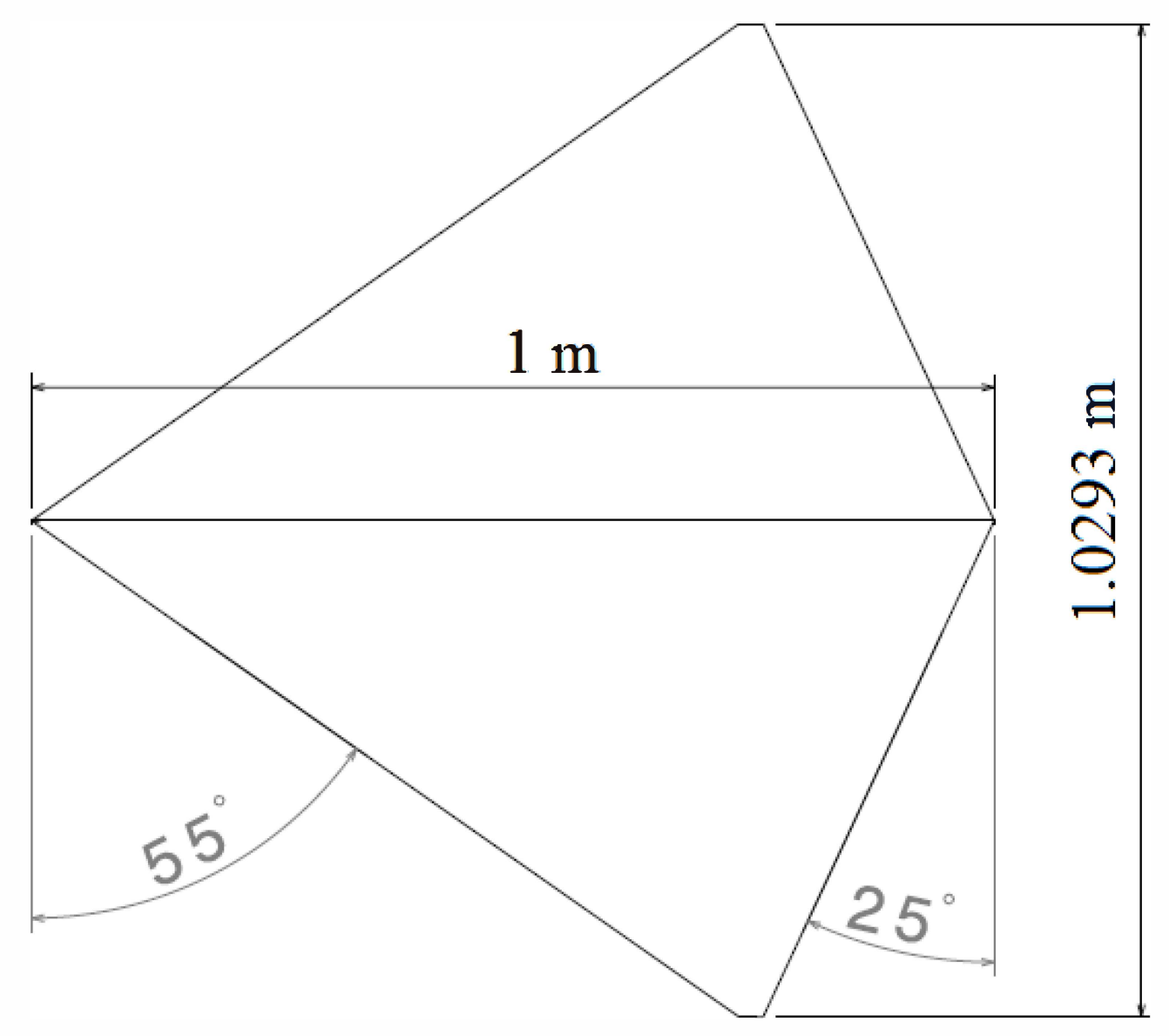

Figure 5. Wing Planform of SAGITTA Demonstrator [2]

- Symmetrical airfoil - NACA64A012 


\section{AVIGLE}

Tomas Bata University in Zlin Faculty of Applied Informatics

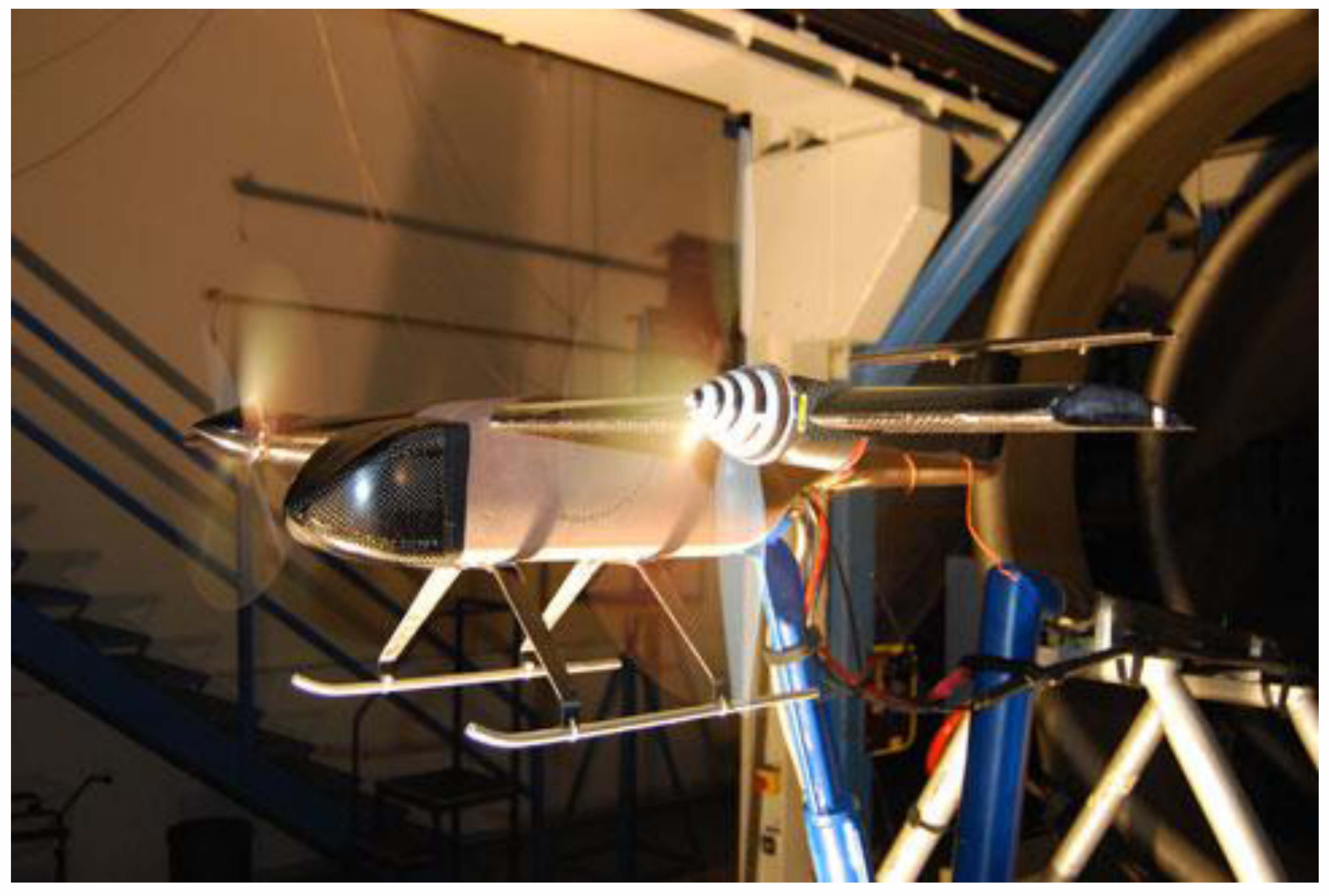

Figure 6. Wind Tunnel Model of AVIGLE UAV [3] 


\section{AVIGLE}

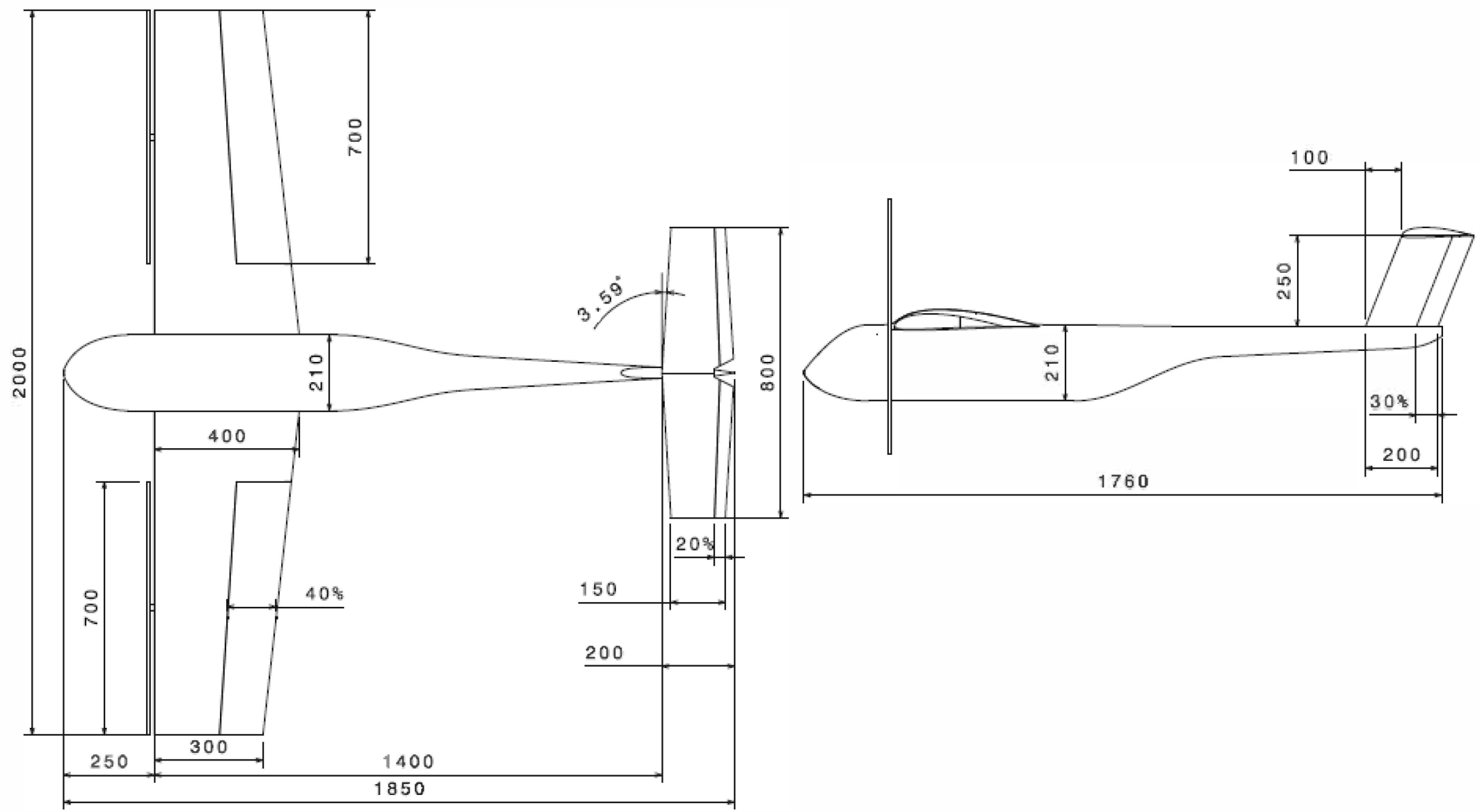

Figure 7. Geometry of AVIGLE UAV [3] 
- 2 mini-UAVs analyzed in 3 CFD applications

$-S U^{2}$

- ROE (Roe's Approximate Riemann Solver)

- JST (Jameson-Schmidt-Turkel)

- OpenFOAM

- Code_Saturne

- Results

- SAGITTA with Boundary Layer

- AVIGLE without Boundary Layer (enGrid crashed)

- SAGITTA without Boundary Layer (for comparison) 


\section{Aerodynamic Analysis - SAGITTA - Lift}

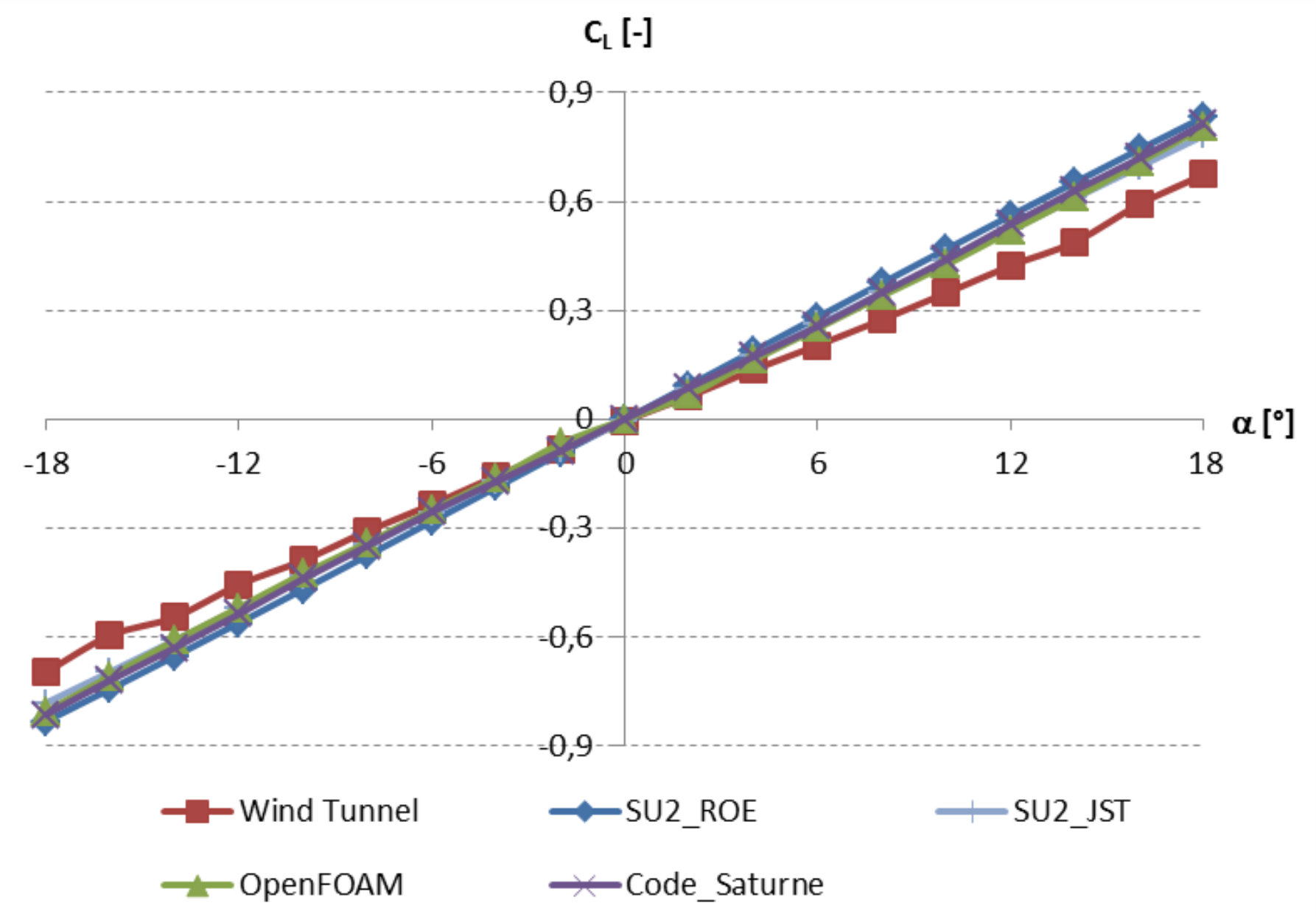

Figure 8. SAGITTA Demonstrator - $C_{L}$ vs. $\alpha$ 


\section{Aerodynamic Analysis - SAGITTA - Drag}

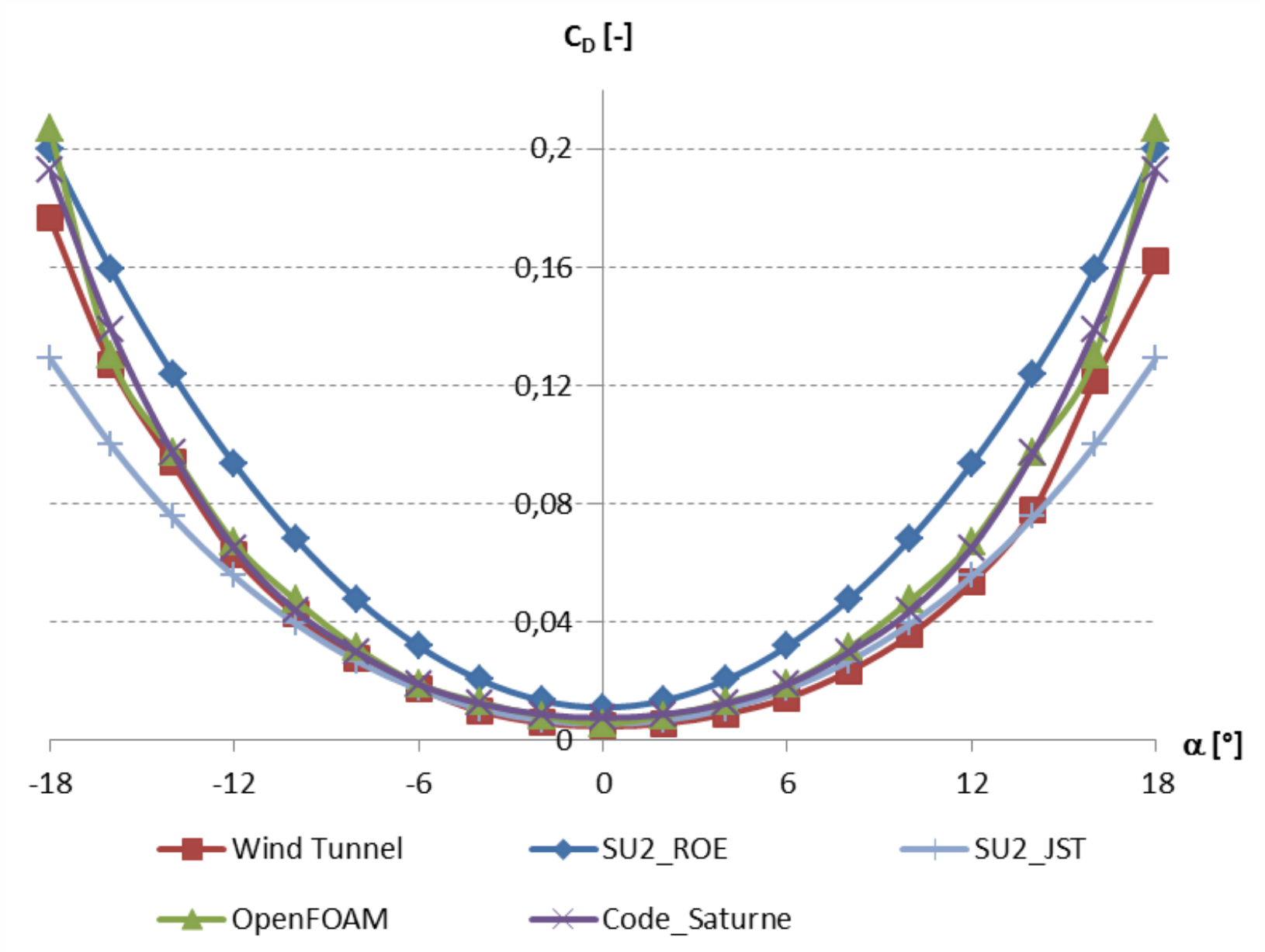

Figure 9. SAGITTA Demonstrator - $C_{D}$ vs. $\alpha$ 


\section{Aerodynamic Analysis - SAGITTA - Lift/Drag}

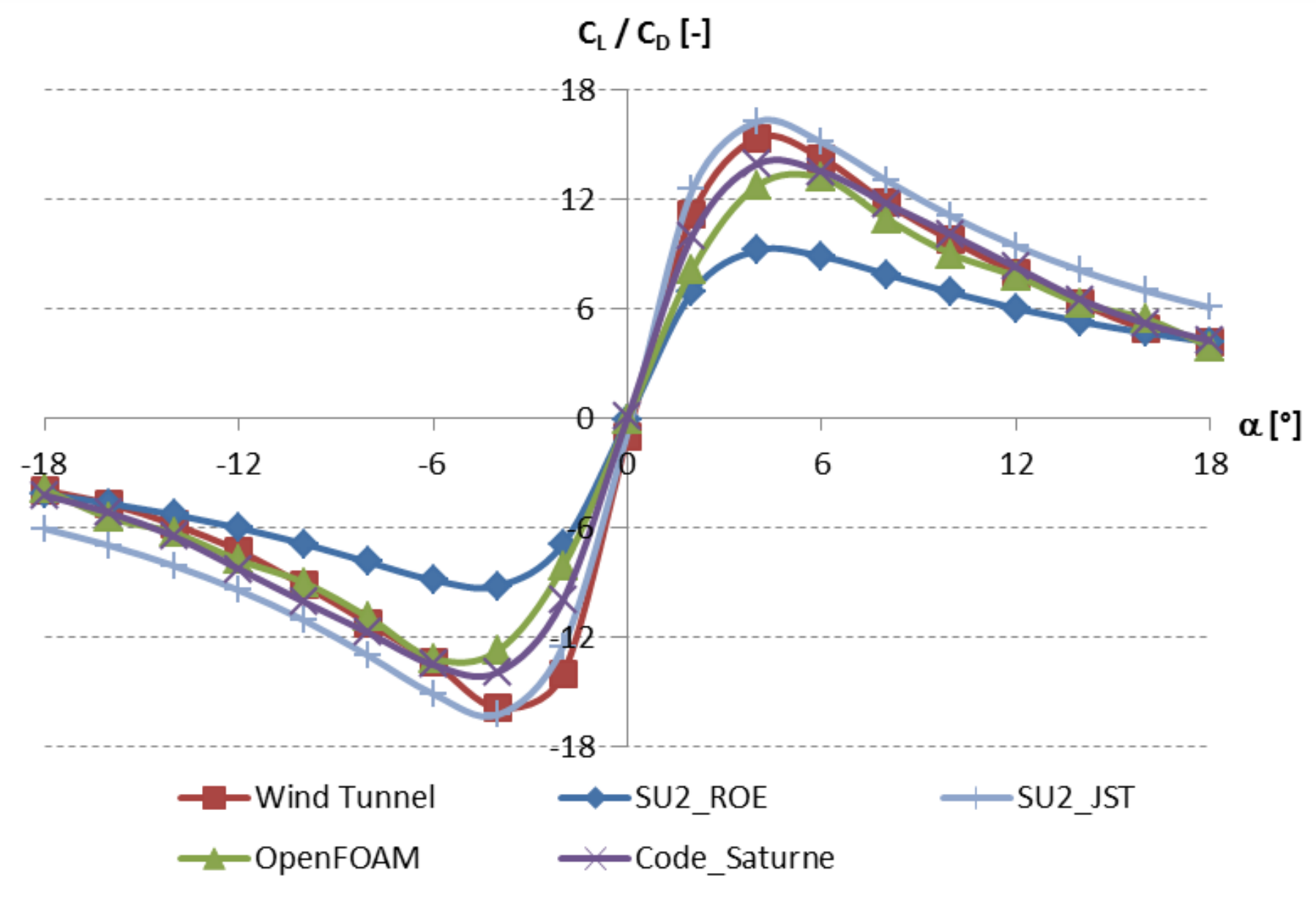

Figure 10. SAGITTA Demonstrator $-C_{L} / C_{D}$ vs. $\alpha$ 


\section{Aerodynamic Analysis - AVIGLE - Lift}

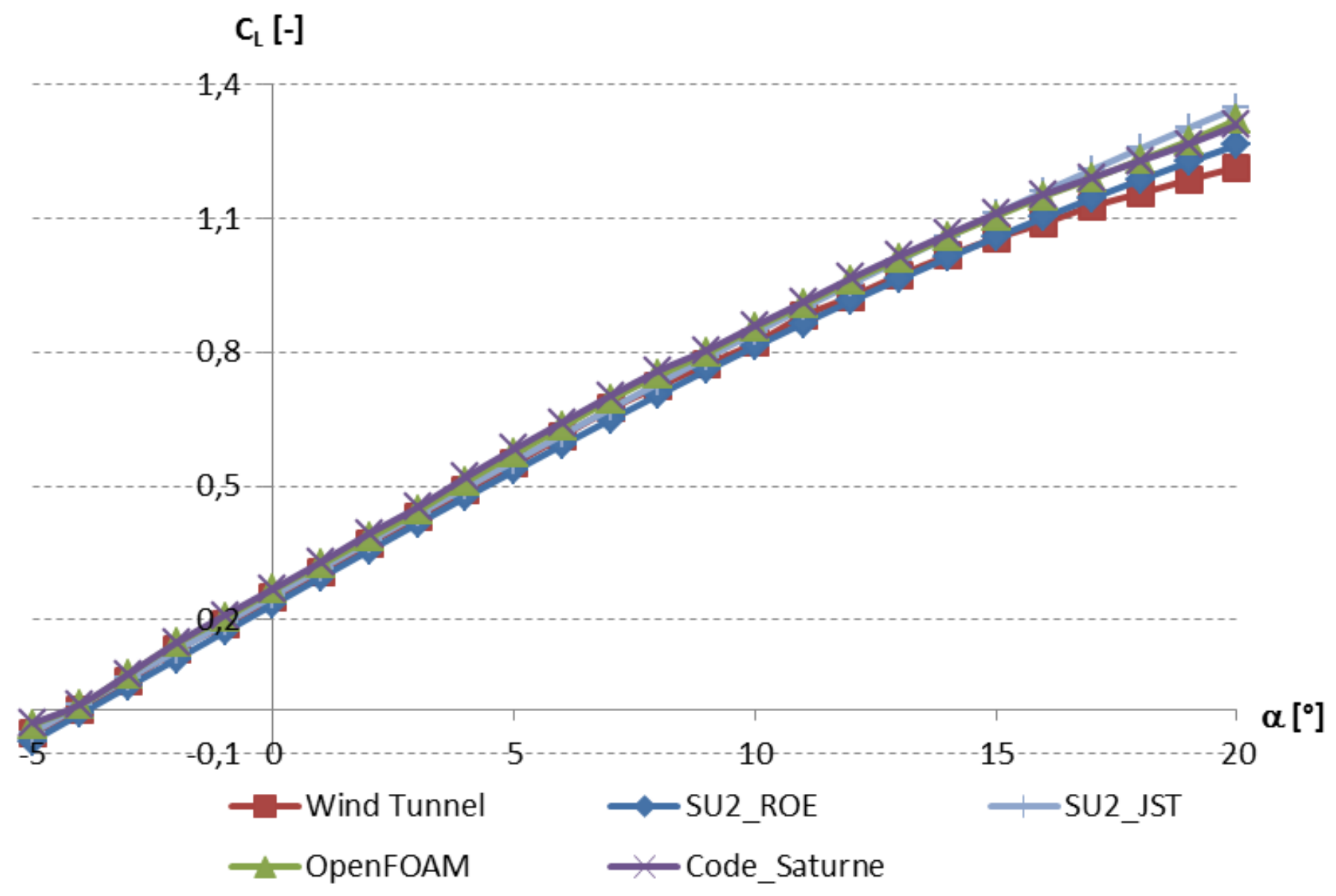

Figure 11. AVIGLE Tiltwing UAV - $C_{L}$ vs. $\alpha$ 


\section{Aerodynamic Analysis - AVIGLE - Drag}

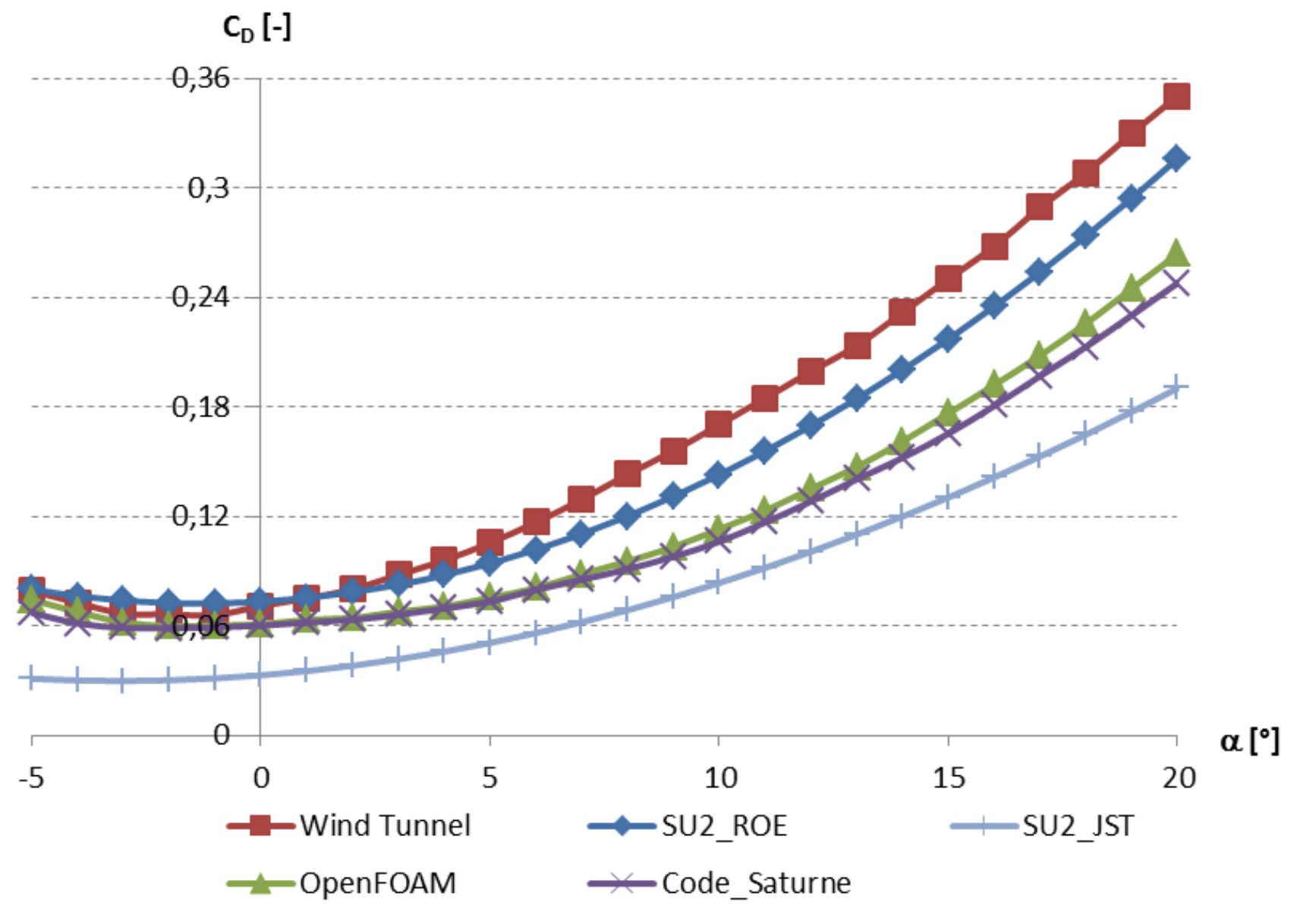

Figure 12. AVIGLE Tiltwing UAV - $C_{D}$ vs. $\alpha$ 


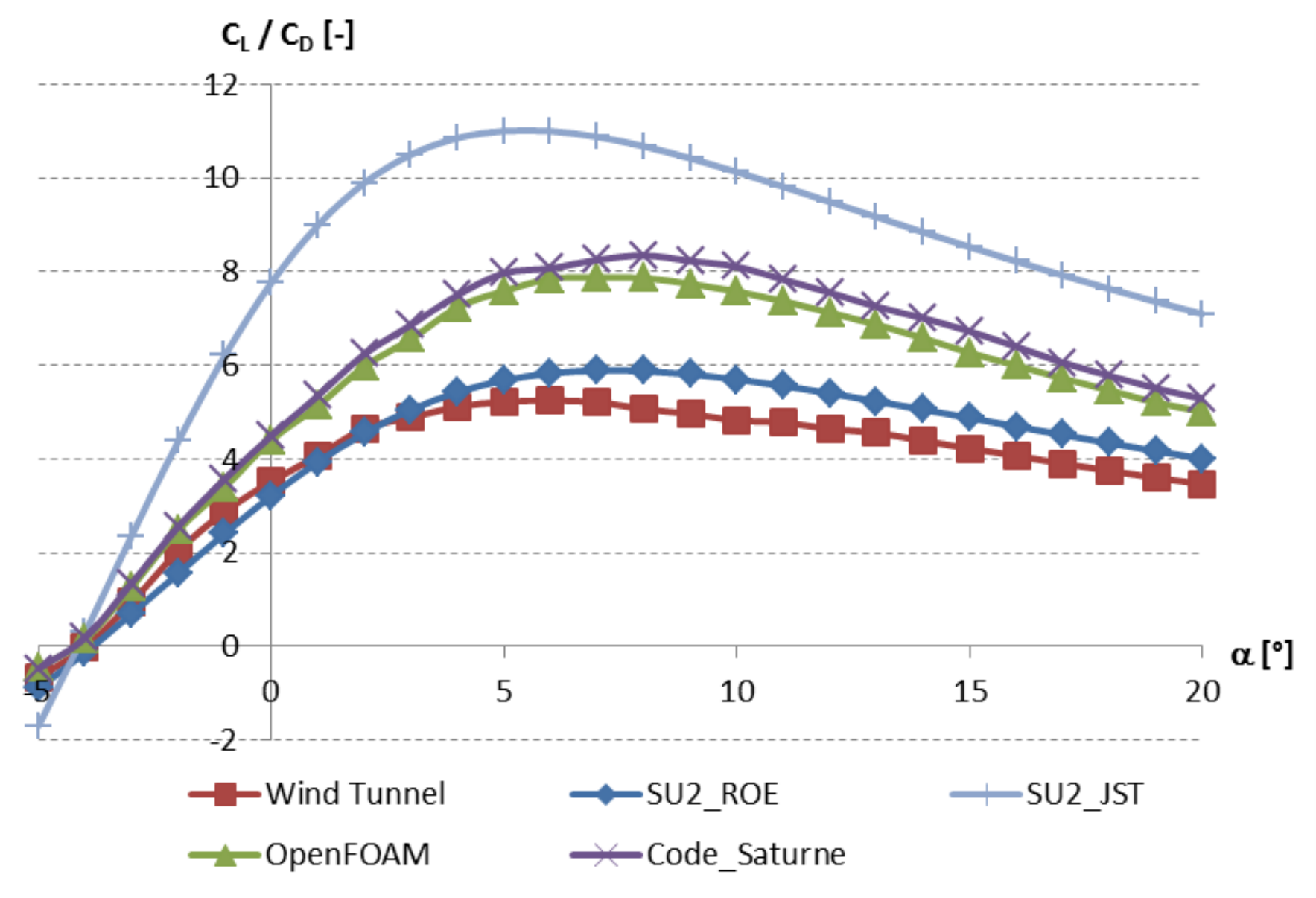

Figure 13. AVIGLE Tiltwing UAV - $C_{L} / C_{D}$ vs. $\alpha$ 


\section{SAGITTA with and without Boundary Layer - Lift}

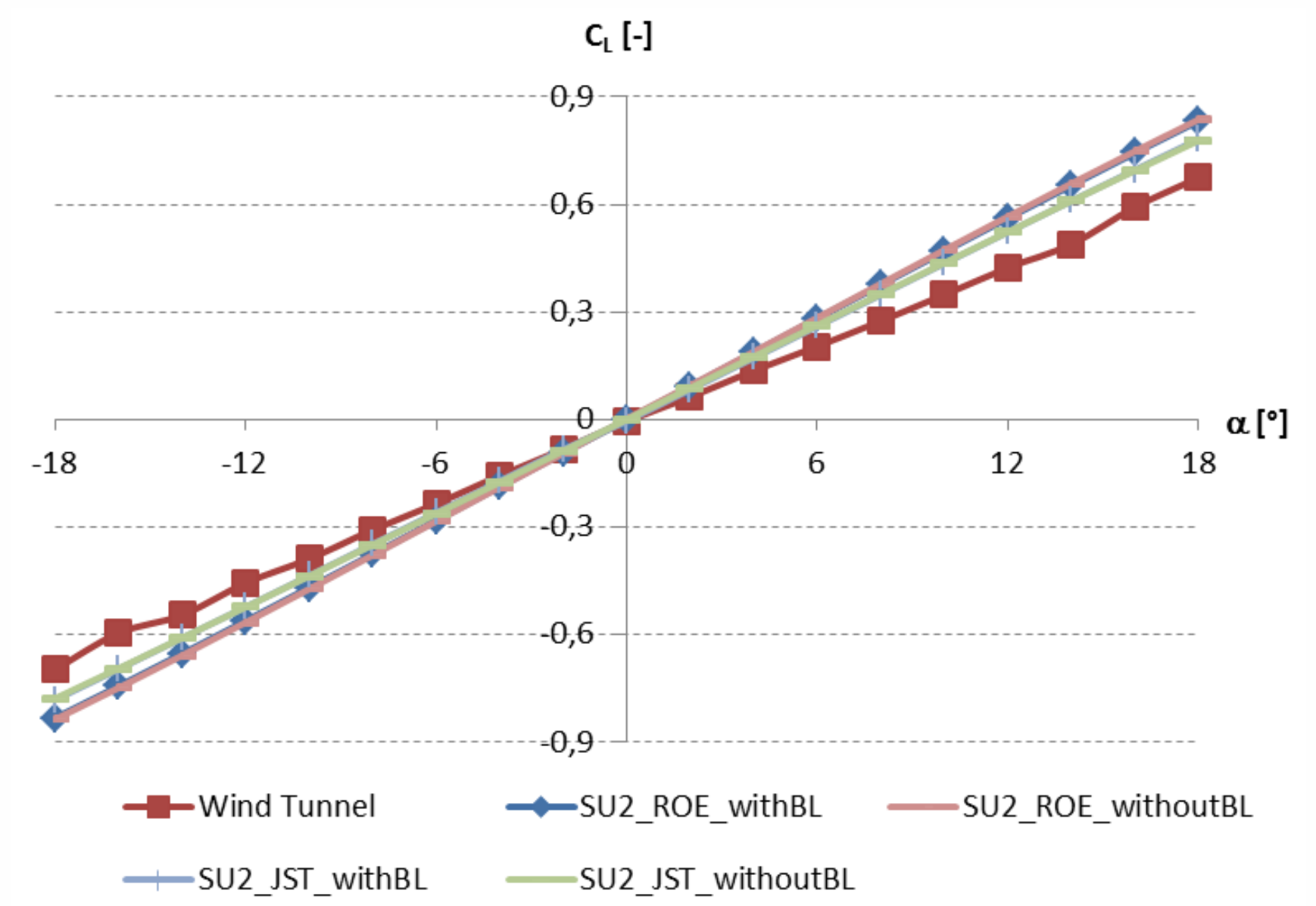

Figure 14. SAGITTA Demonstrator with and without Boundary Layer $-C_{L}$ vs. $\alpha$ 


\section{SAGITTA with and without Boundary Layer - Drag}

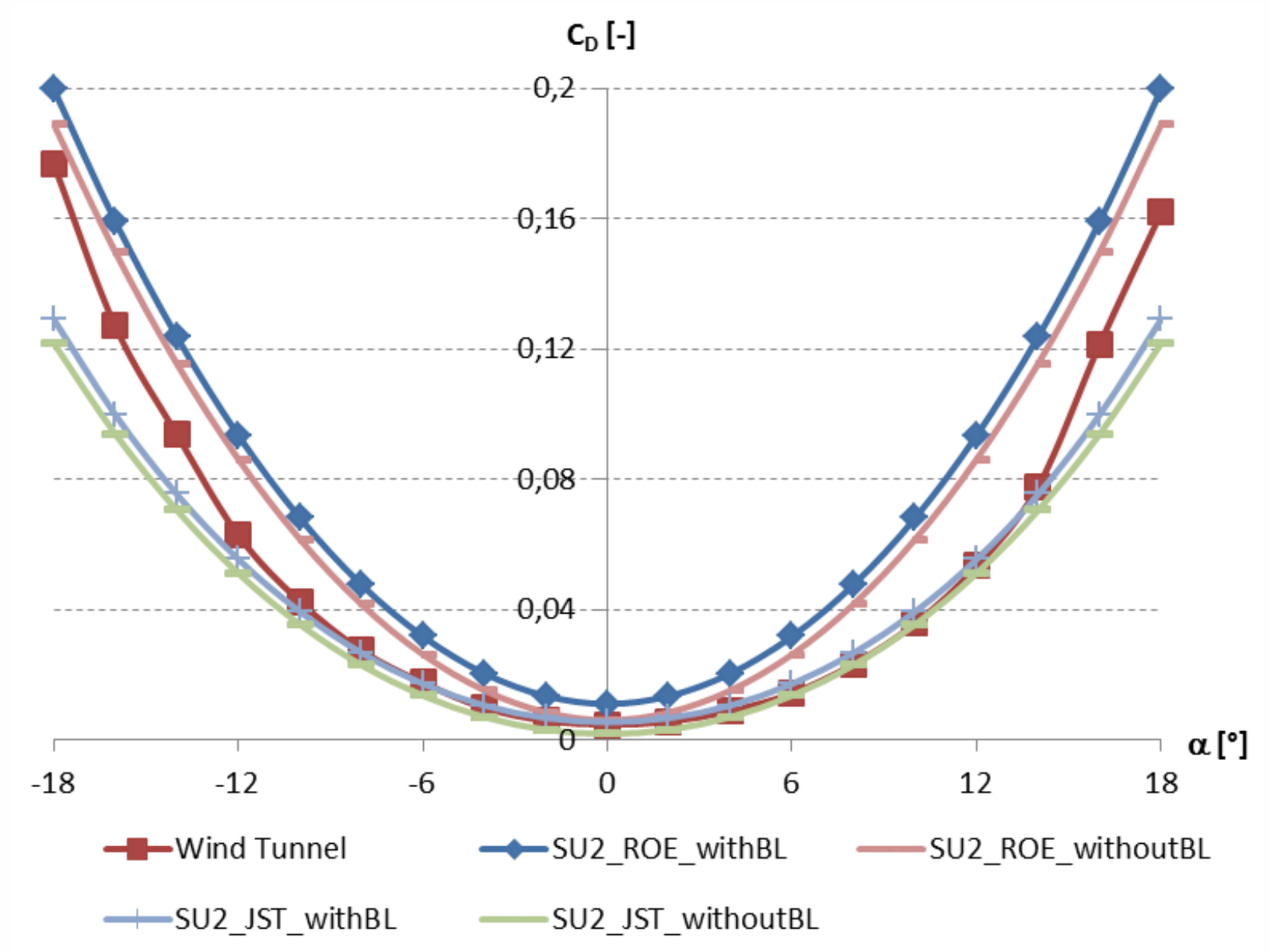

Figure 15. SAGITTA Demonstrator with and without Boundary Layer $-C_{D}$ vs. $\alpha$ 


\section{SAGITTA with and without Boundary Layer - Lift/Drag}

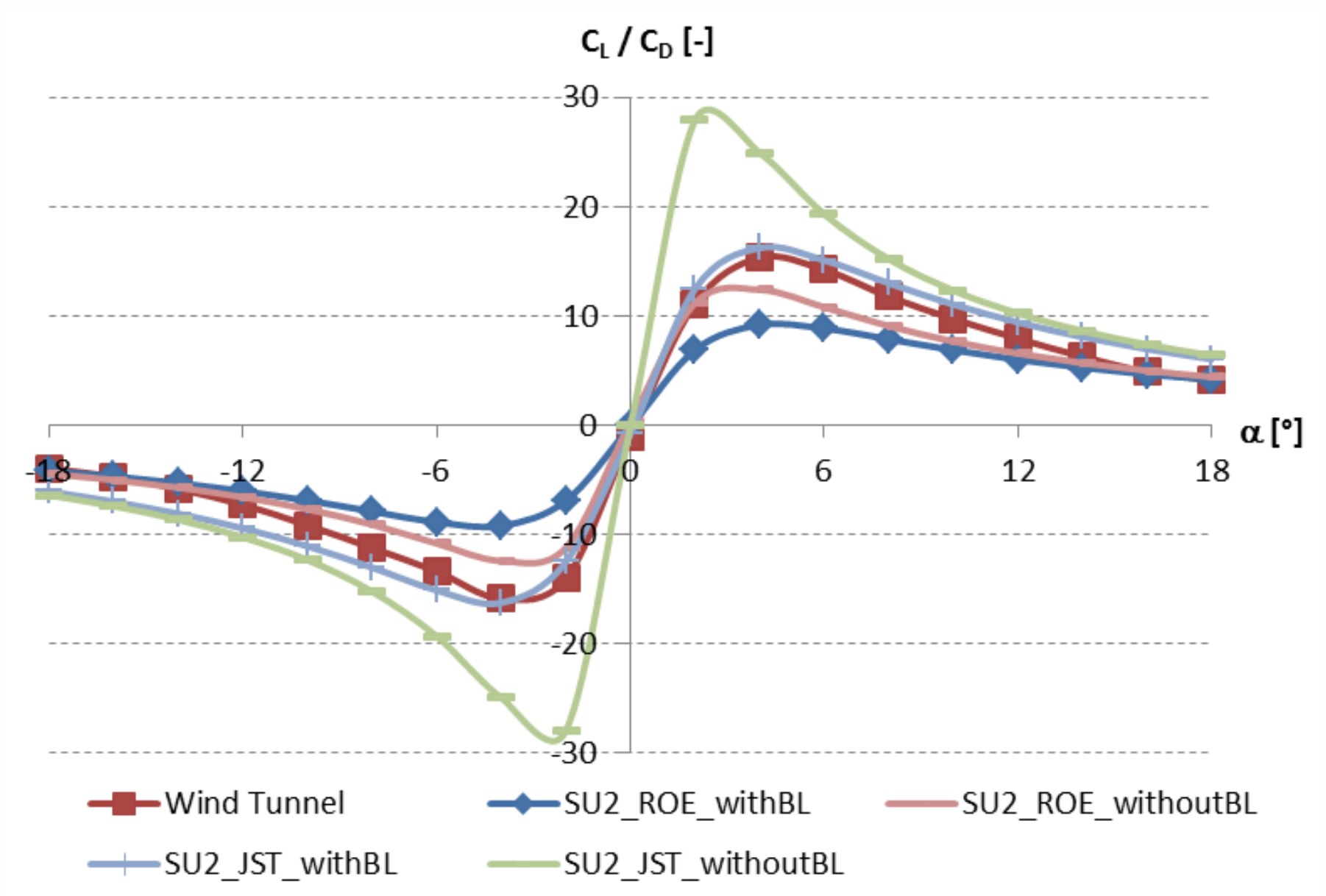

Figure 16. SAGITTA Demonstrator with and without Boundary Layer $-C_{L} / C_{D}$ vs. a 
- CFD results depend on:

- Software/Developers

- Mesh quality of UAV model

- Modeler

- Wind-tunnel results depend on:

- Wind tunnel

- Measuring instruments

- Manufacturing quality of UAV model

- Human factor 
- Open-source CFD software with an appropriate setting and mesh may accurately compute lift and drag coefficients.

- Time vs. Money \& Repeatability

- Similar results from OpenFOAM and Code_Saturne

- $\mathrm{SU}^{2}$

- JST - symmetrical UAVs (or wings) with higher Reynolds number $\left(1.7 \mathrm{e}^{6}\right)$

- ROE - asymmetrical UAVs with lower Reynolds number $\left(2.82 \mathrm{e}^{5}\right)$ 
- Free software

- 2 mini-UAVs analyzed in 3 CFD applications

$-S U^{2}$

- ROE (Roe's Approximate Riemann Solver)

- JST (Jameson-Schmidt-Turkel)

- OpenFOAM

- Code_Saturne

- Results

- SAGITTA with and without Boundary Layer

- AVIGLE without Boundary Layer 


\section{Future Work}

- 3D-printed models -> wind-tunnel tests

- Printed model $\approx$ computer model

- => more precise and objective CFD evaluation

- Results

- PyFR

- HiFiLES 
[1] SAGITTA, June 10, 2015, http://www.unibw.de/lit13 2/Forschung/Projekte/SAGITTA.

[2] Hövelmann, A., C. Breitsamter, 2012, Aerodynamic Characteristic of the SAGITTA Diamond Wing Demonstrator, Deutscher Luft- und Raumfahrtkongress, $14 \mathrm{p}$.

[3] Holsten, J., T. Ostermann, D. Moormann, 2011, Design and wind tunnel tests of a tiltwing UAV, CEAS Aeronautical Journal, vol. 2, no. 1-4, pp. 69-79.

[4] AVIGLE, June 22, 2012, http://www.fsd.rwth-aachen.de/English/Research/Avigle.php

[5] McCormick, Daniel J., May 27, 2002, An Analysis of Using CFD in Conceptual Aircraft Design, Blacksburg, Virginia, USA, Faculty of Virginia Polytechnic Institute and State University, $152 \mathrm{p}$.

[6] Mueller, T. J., J. D. DeLaurier, 2003, Aerodynamics of small vehicles, Annu. Rev. Fluid Mech, vol. 35, no. 1, pp. 89-111, DOI: 10.1146/annurev.fluid.35.101101.161102.

[7] Vogeltanz, Tomáš, 2015 (03/22), A Survey of Free Software for the Design, Analysis, Modelling, and Simulation of an Unmanned Aerial Vehicle, Archives of Computational Methods in Engineering, 66 p., DOI: 10.1007/s11831-015-9147-y.

[8] OpenVSP, August 19, 2015, http://openvsp.org/.

[9] AirfoilTools, 2015, http://airfoiltools.com/. 
[10] enGrid - open-source mesh generation, 2012, http://engits.eu/en/engrid.

[11] Eller, D., Larosterna: about, 2014, http://www.larosterna.com/index.html.

[12] SU2: The Open-Source CFD Code, August 27, 2015,

https://github.com/su2code/SU2/wiki.

[13] OpenFOAM Foundation, OpenFOAM, 2015, http://www.openfoam.org/index.php.

[14] ENGYS Ltd, HELYX-OS: Engys' free-to-download Open Source native GUI for OPENFOAM®, 2015, http://engys.com/products/helyx-os.

[15] EDF R\&D, Welcome to Code_Saturne, 2015, http://code-saturne.org/cms/.

[16] Jin, Wonjin, Yung-Gyo Lee, 2014, Computational Analysis of the Aerodynamic Performance of a Long-Endurance UAV, International Journal of Aeronautical and Space Sciences, vol. 15, no. 4, pp. 374-382, DOI: 10.5139/IJASS.2014.15.4.374..

[17] Uragun, B., December 18-21, 2011, Energy efficiency for unmanned aerial vehicles, 2011 10th International Conference on Machine Learning and Applications, Honolulu, Hawaii, USA, pp. 316-320, DOI: 10.1109/ICMLA.2011.159.

[18] McAlpine, J.D., Computational Fluid Dynamics or Wind Tunnel Modeling?, 5 p., www.envirometrics.com/abstracts/CFDvsWT.pdf. 


\title{
Thank you for your attention
}

\author{
Tomas Bata University in Zlin \\ Faculty of Applied Informatics
}

\section{Do you have any question?}

\author{
vogeltanz@fai.utb.cz
}

